\title{
Expression of apolipoprotein $M$ and its association with adiponectin in an obese mouse model
}

\author{
LIU YANG ${ }^{1}, \mathrm{TIE} \mathrm{LI}^{2}$, SHUIPING ZHAO $^{3}$ and SAIDAN ZHANG ${ }^{4}$ \\ ${ }^{1}$ International Medical Center, Geriatric Department, National Clinical Research Center of \\ Geriatric Diseases, Xiangya Hospital of Central South University, Changsha, Hunan 410008; ${ }^{2}$ Department of Cardiology, \\ Changsha Central Hospital, Changsha, Hunan 410000; ${ }^{3}$ Department of Cardiology, The Second Xiangya Hospital of \\ Central South University, Changsha, Hunan 410011; ${ }^{4}$ Department of Cardiology, Xiangya \\ Hospital of Central South University, Changsha, Hunan 410008, P.R. China
}

Received March 25, 2018; Accepted June 13, 2019

DOI: $10.3892 /$ etm.2019.7755

\begin{abstract}
The aim of the present study was to explore the association between apolipoprotein $\mathrm{M}$ (ApoM) and adiponectin, and the underlying mechanism, via observation of ApoM expression in an obese mouse model. For in vivo experiments, mice were randomly distributed into four groups: Control group, obese group, obese group treated with adiponectin, and normal group treated with adiponectin. Body weight, plasma adiponectin, blood glucose and fasting insulin were measured and visceral adipose tissue was weighed at the end of the experiment. ApoM and transcription factor forkhead box A2 (Foxa2) mRNA expression in the mouse liver was evaluated and the protein level of ApoM detected. For in vitro experiments, an insulin-resistant (IR) hepatic cell model was established by inducing the HepG2 cell line with a high concentration of insulin. Following treatment with adiponectin, changes in ApoM and Foxa 2 mRNA expression and ApoM protein expression were evaluated in the control and IR HepG2 cells. Results demonstrated that compared with the control group, body weight, visceral adipose tissue weight, blood glucose, fasting insulin and insulin-resistance index (HOMA-IR) were significantly increased in the obese group, whilst plasma adiponectin, ApoM mRNA expression, Foxa 2 mRNA expression and ApoM protein in the mouse liver were all significantly decreased. Following intervention with adiponectin in obese mice, blood glucose, insulin and HOMA-IR were significantly decreased, whilst plasma adiponectin, ApoM mRNA expression, Foxa 2 mRNA expression and
\end{abstract}

Correspondence to: Dr Liu Yang, International Medical Center, Geriatric Department, National Clinical Research Center of Geriatric Diseases, Xiangya Hospital of Central South University, 87 Xiangya Road, Changsha, Hunan 410008, P.R. China

E-mail: 15974299612@163.com

Key words: obesity, apolipoprotein $\mathrm{M}$, adiponectin, insulin resistance, mouse model
ApoM protein were all significantly increased. However, no significant difference was observed in visceral adipose tissue weight following the intervention of adiponectin in obese mice. In vitro, in the absence of intervention, ApoM and Foxa 2 mRNA expression and ApoM protein expression were significantly lower in IR HepG2 cells compared with HepG 2 cells. Following intervention with adiponectin on IR HepG 2 cells, ApoM and Foxa 2 mRNA expression and ApoM protein expression were significantly increased. However, the intervention did not have any effect on HepG2 cells. In conclusion, intervention with adiponectin elevated ApoM mRNA expression, potentially via relieving IR and upregulating Foxa2 mRNA expression.

\section{Introduction}

Obesity has become a major global healthcare issue with increasing prevalence across the world. The condition is highly associated with dyslipidemia, metabolic syndrome, type 2 diabetes, hypertension, hyperuricemia and many other diseases, and is an independent risk factor for mortality and lower quality of life for patients $(1,2)$. Obesity is often considered to result in decreased high-density lipoprotein-cholesterol (HDL-C), whilst the level of HDL-C is negatively correlated with the incidence of coronary heart disease (3). Numerous studies have demonstrated that HDL has a protective effect on the arterial wall therefore apolipoproteins related to the metabolism of HDL are increasingly gaining attention. A recently discovered apolipoprotein, ApoM, is an important part of HDL and contributes considerably to the metabolism of HDL-C (4). Currently, it remains unclear whether HDL-C deficiency in the blood is associated with ApoM level. Furthermore, obesity is typically accompanied by hyperleptinemia and hyperadiponectinemia, and certain studies have revealed that leptin is associated with ApoM. However, it has not yet been reported whether there is an association between adiponectin and ApoM. Therefore, the present study investigated the underlying mechanism of blood HDL-C deficiency during obesity via detection of ApoM expression in obese mice, and also the possible association between ApoM and adiponectin. 
Insulin resistance (IR) is closely associated to obesity $(5,6)$ and can aggravate the progress of the condition whilst promoting the development of diabetes, hyperlipidemia and hypertension. IR is characterized by the insensitivity of the target tissue to insulin, and also decreased uptake and utilization of glucose in peripheral tissues. The liver and peripheral tissues (fat and muscle) are the main sites of IR. HepG2 cells are derived from hepatocytes and retain many of the properties of hepatocytes. Following co-incubation with high concentrations of insulin, HepG2 cells exhibit the functional defects of insulin receptors and post-receptors (7-10). IR induced by a high concentration of insulin in HepG2 cells is an ideal cell model for studying this condition (11-14). Presently, adiponectin is the only adipocyte-derived cytokine found to have a protective effect on humans and can significantly improve IR (15). In order to explore the mechanism of adiponectin on ApoM, the effects of adiponectin on IR in obese mice and IR HepG2 cells were investigated.

\section{Materials and methods}

Materials and reagents. C57BL/6N male mice (at 3 weeks of age) were purchased from the Shanghai Experimental Animal Center of the Chinese Academy of Sciences. The human hepatoblastoma cell line HepG2 was kindly provided by the Xiangya School of Medicine, Central South University (Changsha, China). Fluorescent quantitative polymerase chain reaction (PCR) kit was purchased from Promega Corporation. The antibody for ApoM detection was obtained from Abcam (cat. no. ab66379) and an insulin ELISA kit (cat. no. 10-1247-01) was obtained from Mercodia. All PCR primers were synthesized by Aoke Biotechnology Company, Ltd. (Table I). TRIzol ${ }^{\circledR}$ reagent was purchased from Invitrogen (Thermo Fisher Scientific, Inc.). A plasma insulin detection kit was purchased from Mercodia. Recombinant mouse adiponectin was obtained from Abcam (cat. no. ab54483).

Animal grouping and treatment. The Ethics Committee of the Xiangya Hospital of Central South University reviewed and approved this study. A total of $32 \mathrm{C} 57 \mathrm{BL} / 6 \mathrm{~N}$ male mice (3 weeks of age), initially weighing $\sim 9.5 \mathrm{~g}$, were observed for 1 week whilst housed in single-cages (12-h light/dark cycle; temperature $24-28^{\circ} \mathrm{C}$, relative humidity $60-75 \%$ ) and fed individually. Mice were then randomly and evenly distributed into four groups: The control group where mice were fed with a regular diet for 12 weeks; the obese group where mice were fed with a fat-rich diet (from Research Diets D12492) for 12 weeks; the obese group with intervention where mice were fed with a fat-rich diet (Research Diets D12492) for 12 weeks then treated with adiponectin for a further 7 days; and the normal group with intervention where mice were fed with a regular diet for 12 weeks followed by 7 days of treatment with adiponectin. The recombinant mouse adiponectin was dissolved in PBS reaching a final concentration of $0.5 \mu \mathrm{g} / \mu \mathrm{l}$. Adiponectin was administered to mice intraperitoneally (IP) at $1.5 \mathrm{mg} / \mathrm{kg}$ body weight once per day for 7 days. It was administered at a speed of $10 \mu \mathrm{l} / \mathrm{min}$ from 17:00. Mice had free access to food and water. At the end of the experiment, body weight, fasting blood glucose and plasma adiponectin levels were measured following which all mice were sacrificed under anesthesia to minimize suffering. Mice were anesthetized by IP injection of sodium pentobarbital $(2 \%, 40 \mathrm{mg} / \mathrm{kg})$ and executed by cardiac puncture. Liver tissue was isolated and preserved in liquid nitrogen. Visceral adipose tissue weight was obtained by measuring the wet weight of the epididymidis and adipose tissue surrounding the kidney with an electronic balance (the adhering tissue fluid was removed by suction with filter paper). Plasma insulin and adiponectin levels were determined by ELISA. Mice were fasted for 6-8 $\mathrm{h}$ then blood samples were obtained from the caudal vein to determine fasting plasma glucose (FPG) level with a One-Touch blood glucose meter (LifeScan, Inc.) The formula for calculation of IR index was: IR index [Homeostatic model assessment (HOMA)-IR]=FPG $\mathrm{x}$ fasting insulin/22.5.

Animal diets. The basal diet was prepared by the Animal Laboratory of Xiangya School of Medicine, providing energy of $15 \mathrm{~kJ} / \mathrm{g}$, with a contribution of $23 \%$ protein, $65 \%$ carbohydrates and $12 \%$ fat. The fat-rich diet (D12492) was provided by Research Diets Company, providing energy of $22 \mathrm{~kJ} / \mathrm{g}$, with a contribution of $20 \%$ protein, $20 \%$ carbohydrates and $60 \%$ fat.

Maintenance and subculture of the HepG2 cell line. The HepG2 cell line was cultured with Dulbecco's modified Eagle's medium (Gibco; Thermo Fisher Scientific, Inc.), supplemented with 10\% fetal bovine serum (Tian Hang Biotechnology Company) and $1.0 \times 10^{5} \mathrm{U} / 1$ penicillin-streptomycin and incubated under standard conditions $\left(37^{\circ} \mathrm{C}, 5 \% \mathrm{CO}_{2}\right)$. Medium was replaced every other day and subculture was performed using $0.25 \%$ trypsin to detach cells from the culture vessel.

The liver is the organ with highest metabolic activity in humans and is responsible for a variety of important functions, such as lipid metabolism, therefore it is reasonable to employ hepatic cells in studies on lipid metabolism in vitro. It is extremely difficult to obtain normal hepatic cells under physiological conditions, and normal human hepatic cells usually exhibit marked individual differences that make it hard to achieve stable subcultures. For example, the human hepatic cell line HL-7702, which is derived from normal human hepatic tissue, shows dramatic biological differences to primary hepatic cell cultures. Human hepatic cell lines, L-02, LX-1 and LX-2 could be regarded as good representatives of the human liver (16); however, they possess defective regeneration abilities which makes them less suitable options for in vitro experiments (17). The HepG2 cell line, derived from human hepatoblastoma, possesses a strong regenerative ability as well as the majority of all liver-related functions $(18,19)$, and could effectively mimic the in vivo environment for lipid metabolism. Therefore, the HepG2 cell line was selected to perform all in vitro experiments.

Establishment of IR in HepG2 cell model. Studies typically use glucose consumption as a criterion to determine whether IR is successful (20-22). HepG2 cells at logarithmic growth stage were digested, the cell density adjusted to $5 \times 10^{4}$ cells $/ \mathrm{ml}$ using serum-containing medium, then $200 \mu \mathrm{l}$ cell suspension was added to each well of a 96-well culture plate. Samples were divided into either control group (no insulin stimulation, normal cultured cells) or IR model group. Following monolayer adherence, serum-containing medium was discarded and 
Table I. Sequences of primers used for reverse transcription-quantitative polymerase chain reaction.

\begin{tabular}{|c|c|c|c|}
\hline Gene & Resource & Primer & Sequence \\
\hline \multirow[t]{2}{*}{ ApoM } & $\mathrm{Mu}$ & Forward & 5'-CAGTGCCCTGAGCACAGTCAA-3' \\
\hline & & Reverse & 5'-GCTGCTCCCGCAATAAAGTACC-3' \\
\hline \multirow[t]{2}{*}{ ApoM } & Hs & Forward & 5'-CTGACAACTCTGGGCGTGGA-3' \\
\hline & & Reverse & 5'-CAGAGCCAGCAGCCATATTGAA-3' \\
\hline \multirow[t]{2}{*}{ Foxa2 } & $\mathrm{Mu}$ & Forward & 5'-GTCGTCCGAGCAGCAACATC-3 \\
\hline & & Reverse & 5'-GGGTAGTGCATGACCTGTTCGTAG-3' \\
\hline \multirow[t]{2}{*}{ Foxa2 } & Hs & Forward & 5'-CGTCCGACTGGAGCAGCTACTAT-3 \\
\hline & & Reverse & 5'-CGGCGTTCATGTTGCTCAC-3' \\
\hline \multirow[t]{2}{*}{ GAPDH } & $\mathrm{Mu}$ & Forward & 5'-ACAGCAACAGGGTGGTGGAC-3' \\
\hline & & Reverse & 5'-TTTGAGGGTGCAGCGAACTT-3' \\
\hline \multirow[t]{2}{*}{ GAPDH } & Hs & Forward & 5'-CCATGTTCGTCATGGGTGTGAACCA-3' \\
\hline & & Reverse & 5'-GCCAGTAGAGGCAGGGATGATGTTC-3' \\
\hline
\end{tabular}

ApoM, apolipoprotein M; Foxa2, forkhead box A2; Mu, Mus musculus; Hs, Homo sapiens.

cells washed with PBS. Serum-free medium was added to the control group and serum-free medium containing $10^{-7} \mathrm{~mol} / \mathrm{l}$ insulin was added to the model group. The supernatant was removed from the culture medium $24 \mathrm{~h}$ following treatment, then glucose content was measured using the glucose oxidase assay kit (Zhongbei Biotechnology Co., Ltd.). The glucose consumption was determined by calculating the difference between glucose content in the cell culture medium without cells and glucose content in the experimental group.

Cell groups and interventions. Adiponectin was used as an intervention in IR HepG2 cells and HepG2 cells for $24 \mathrm{~h}$. All chemicals were dissolved in dimethyl sulphoxide (DMSO) and the final concentration of DMSO in media was maintained at $0.1 \%(\mathrm{v} / \mathrm{v})$. The treatment groups were as follows: Control group, which was treated with $0.1 \%$ DMSO; IR group, which was treated with $10^{-7} \mathrm{~mol} / \mathrm{l}$ insulin; adiponectin intervention IR group, which was treated with a combination of $10^{-7} \mathrm{~mol} / \mathrm{l}$ insulin and $30 \mu \mathrm{g} / \mathrm{ml}$ adiponectin; and adiponectin intervention group, which was treated with $30 \mu \mathrm{g} / \mathrm{ml}$ adiponectin. All treatments lasted $24 \mathrm{~h}$, then glucose consumption and ApoM and forkhead box A2 (Foxa2) gene expression were evaluated in each group.

Reverse transcription-quantitative polymerase chain reaction $(R T-q P C R)$. Total RNA was extracted from mouse liver and other tissues using TRIzol (Invitrogen; Thermo Fisher Scientific, Inc.) according to the manufacturer's protocol. RNA was inspected under ultraviolet light following formaldehyde-denatured agarose gel electrophoresis for $10 \mathrm{~min}$ (stained with ethidium bromide, buffered with 1X MOPS, applied with constant voltage of $5 \mathrm{~V} / \mathrm{cm}$ ). The synthesis of cDNA was performed according to the Avian Myeloblastosis Virus reverse transcriptase protocol (Promega Corporation), and purified via the PAGE method. GAPDH mRNA was employed as the loading control to ensure even loading of all RNA samples. The buffer for SYBR Green Real Time PCR and reaction conditions were selected based on the manufacturer's protocol for the Real Time PCR Master kit (Promega Corporation): $50^{\circ} \mathrm{C}$ for $2 \mathrm{~min}, 95^{\circ} \mathrm{C}$ for $10 \mathrm{~min}$ for the first cycle, and $95^{\circ} \mathrm{C}$ for $15 \mathrm{sec}, 61^{\circ} \mathrm{C}$ for $45 \mathrm{sec}$ and $61^{\circ} \mathrm{C}$ for $10 \mathrm{sec}$ for 40 cycles, until the reaction ended. The transcription level of target genes (Table I) in all samples was normalized to the internal reference gene GAPDH and analyzed via the $2^{-\Delta \Delta C q}$ method (23).

Detection of ApoM expression by western blot analysis. Proteins were extracted using RIPA lysis buffer (Shanghai Biyuntian Biotechnology Co., Ltd.) from cultured cells or mouse livers. Proteins were then determined via a BCA assay. For each sample, $50 \mu \mathrm{g}$ of total protein was diluted in loading buffer, boiled in a $100^{\circ} \mathrm{C}$ water bath for $10 \mathrm{~min}$ then stored on ice. Protein samples were separated by $6 \%$ SDS-PAGE then transferred onto polyvinylidene difluoride membranes and blocked with 5\% skimmed milk for $2 \mathrm{~h}$. Then the membrane was incubated with primary antibody ApoM (1:500) at $4^{\circ} \mathrm{C}$ overnight. Membranes were washed in a mixture of tris-buffered saline and Polysorbate (TBST) three times (10 $\mathrm{min} /$ wash) then incubated in horseradish peroxidase-conjugated secondary antibody $(1: 2,000)$ for $1 \mathrm{~h}$ at RT then washed in TBST three more times. X-ray films were used to capture the signals from membranes that had been processed using a western blot chemiluminescence detection reagent kit (Pierce; Thermo Fisher Scientific, Inc.) The bands on films were analyzed with TINA 2.09 image processing software (Raytest). Densitometry analysis was performed with the control group set as $100 \%$ for comparisons with bands of other groups and for related quantitative analysis. $\beta$-actin was used as loading control.

Statistical analysis. All experimental data are presented as the mean \pm standard deviation and analyzed with the software SPSS 15.0 (SPSS, Inc.). Comparisons among groups were evaluated using one-way analysis of variance and Student-Newman-Keuls test. $\mathrm{P}<0.05$ was considered to indicate significant difference. 
Table II. Comparisons of body weight, visceral adipose tissue weight, levels of blood glucose, insulin, HOMA-IR and plasma adiponectin.

\begin{tabular}{lrrrr}
\hline Variables & Control & Obesity & Obesity with intervention & Control with intervention \\
\hline Body weight by the end of 12th week $(\mathrm{g})$ & $29.21 \pm 1.51$ & $42.37 \pm 1.42^{\mathrm{a}}$ & $42.67 \pm 1.33^{\mathrm{a}}$ & $28.92 \pm 1.32$ \\
Visceral adipose tissue weight $(\mathrm{g})$ & $0.55 \pm 0.02$ & $1.81 \pm 0.50^{\mathrm{a}}$ & $1.79 \pm 0.32^{\mathrm{a}}$ & $0.54 \pm 0.03$ \\
Blood glucose (mmol/l) & $8.69 \pm 1.13$ & $13.66 \pm 1.27^{\mathrm{a}}$ & $11.23 \pm 1.23^{\mathrm{a}, \mathrm{b}}$ & $8.50 \pm 1.17$ \\
Plasma insulin (ng/ml) & $0.35 \pm 0.02$ & $0.75 \pm 0.05^{\mathrm{a}}$ & $0.60 \pm 0.07^{\mathrm{a}, \mathrm{b}}$ & $0.33 \pm 0.02$ \\
HOMA-IR & $0.13 \pm 0.01$ & $0.45 \pm 0.03^{\mathrm{a}}$ & $0.30 \pm 0.05^{\mathrm{a}, \mathrm{b}}$ & $0.12 \pm 0.01$ \\
Adiponectin (mg/l) & $2.11 \pm 0.03$ & $1.32 \pm 0.07^{\mathrm{a}}$ & $1.97 \pm 0.03^{\mathrm{b}}$ & $3.19 \pm 0.07^{\mathrm{a}}$ \\
\hline
\end{tabular}

${ }^{\mathrm{a}} \mathrm{P}<0.05$ vs. control group; ${ }^{\mathrm{P}}<0.05$ vs. obese group. HOMA-IR, homeostatic model assessment-insulin resistance.

\section{Results}

Comparisons of body weight, visceral adipose tissue weight, blood glucose, insulin, HOMA-IR and plasma adiponectin amongst groups. At the end of week 12, body weight and visceral adipose tissue weight of the obese group and the obese group with intervention were significantly higher compared with the control group $(\mathrm{P}<0.05$; Table II). The levels of blood glucose, insulin and HOMA-IR were all elevated compared with the control group which indicated that the continuous fat-rich diet could lead to elevated blood glucose, hyperinsulinemia and IR. The adiponectin level in the obese group was markedly decreased when compared with the control group $(\mathrm{P}<0.05$; Table II). Intervention with adiponectin in obese mice was associated with lower levels of blood glucose, insulin and HOMA-IR compared with the obese group ( $\mathrm{P}<0.05$; Table II). Plasma adiponectin in the obese group with intervention was significantly higher compared with the obese group, however there was no significant difference in visceral adipose tissue weight $(\mathrm{P}>0.05$; Table II). Intervention in the control group led to elevated plasma adiponectin level compared with the control group without intervention, however no significant differences were observed in terms of visceral adipose tissue weight, levels of blood glucose, insulin and HOMA-IR (P>0.05; Table II).

Levels of ApoM $m R N A$ and protein in mouse livers. ApoM mRNA expression (Fig. 1) and protein levels (Fig. 2) in the obese group were markedly decreased when compared with the control group $(\mathrm{P}<0.05)$. Following intervention with adiponectin, ApoM mRNA expression (Fig. 1) and protein levels (Fig. 2) were elevated in the obese group with intervention, compared with the obese group. There was no significant difference between the control group with intervention and the control group $(\mathrm{P}>0.05)$.

Foxa2 mRNA expression levels in mouse livers. Compared with the control group, the Foxa 2 mRNA expression levels were significantly lower in the obese group ( $\mathrm{P}<0.05$; Fig. 3$)$. Following intervention with adiponectin, the Foxa2 mRNA expression level was markedly elevated in the obese group with intervention compared with the obese group $(\mathrm{P}<0.05$; Fig. 3). However, there was no significant difference in Foxa2 mRNA expression levels between the control group and the control group with intervention ( $\mathrm{P}>0.05$; Fig. 3 ).

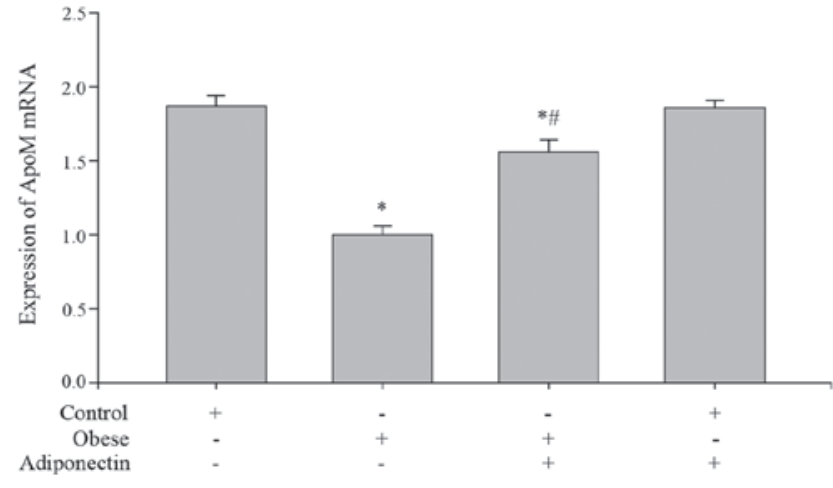

Figure 1. ApoM mRNA expression levels in mouse livers. Following intervention with adiponectin, ApoM mRNA expression was elevated compared with the untreated obese group. There was no significant difference between the control group with intervention and the control group. ${ }^{*} \mathrm{P}<0.05$ vs. control; ${ }^{\#} \mathrm{P}<0.05$ vs. obese group. ApoM, apolipoprotein $\mathrm{M}$.

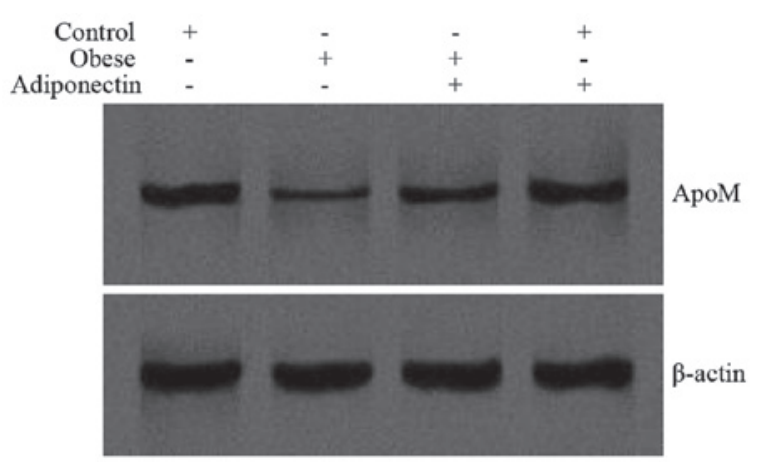

Figure 2. ApoM protein levels in mouse livers. Following intervention with adiponectin, ApoM protein levels were elevated in the obese group compared with the untreated obese group. There was no significant difference between the control group with intervention and the control group. $\beta$-actin was used as loading control. ApoM, apolipoprotein M.

Levels of ApoM mRNA and protein in HepG2 cells. Compared with the control group, the levels of ApoM mRNA expression and protein were significantly decreased in the IR group $(\mathrm{P}<0.05$; Figs. 4 and 5). Following intervention, the levels of ApoM mRNA expression and protein were both significantly increased in the adiponectin intervention IR group compared with the IR group $(\mathrm{P}<0.05$; Figs. 4 and 5$)$. There 


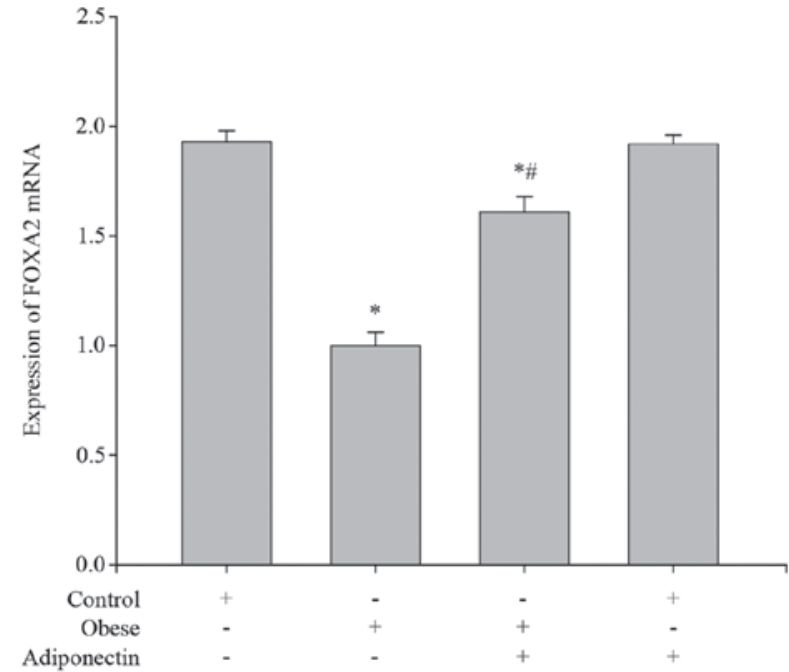

Figure 3. Foxa2 mRNA expression levels in mouse livers. Compared with the control group, the Foxa2 mRNA expression levels were significantly lower in the untreated obese group. Following intervention with adiponectin, the Foxa2 mRNA expression levels were markedly elevated compared with the untreated obese group. There was no significant difference in Foxa2 mRNA expression levels between the control group and the control group with intervention. " $\mathrm{P}<0.05$ vs. control; ${ }^{\#} \mathrm{P}<0.05$ vs. obese group. Foxa2, forkhead box A2.

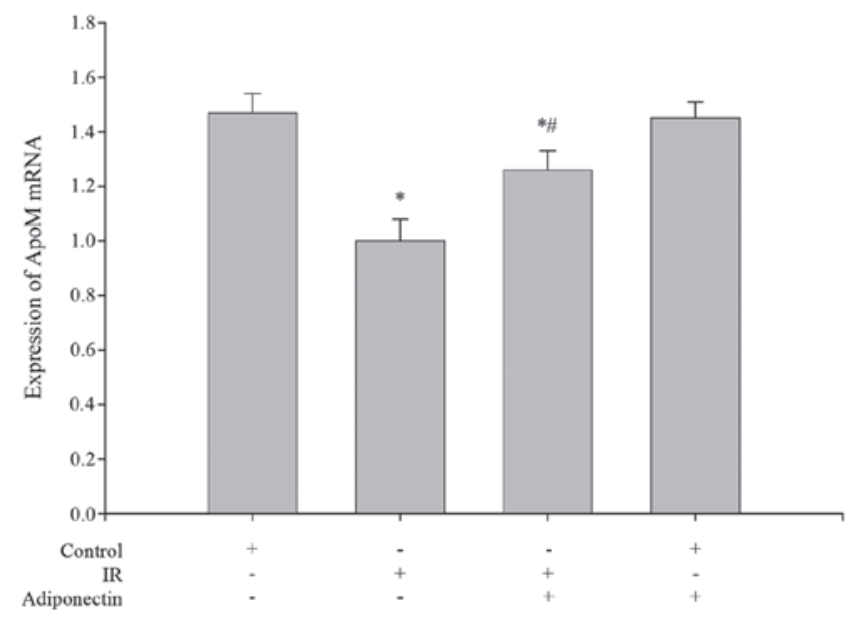

Figure 4. ApoM mRNA expression levels in HepG2 cells. Compared with the control group, ApoM mRNA expression was significantly decreased in the IR group. Following intervention with adiponectin, ApoM mRNA expression was significantly increased compared with the untreated IR group. There was no significant difference in ApoM mRNA expression between the control group and the adiponectin intervention group. ${ }^{*} \mathrm{P}<0.05$ vs. control; ${ }^{~} \mathrm{P}<0.05$ vs. IR group. ApoM, apolipoprotein M; IR, insulin resistance.

were no significant differences in ApoM mRNA expression or protein levels between the control group and the adiponectin intervention group ( $\mathrm{P}>0.05$; Figs. 4 and 5).

Foxa2 mRNA expression levels in HepG2 cells. Compared with the control group, the Foxa2 mRNA expression levels were significantly decreased in the IR group $(\mathrm{P}<0.05$; Fig. 6$)$. Following treatment with adiponectin, the Foxa2 mRNA expression levels were significantly increased in the adiponectin intervention IR group compared with the IR group $(\mathrm{P}<0.05$; Fig. 6). However, no significant difference in Foxa2

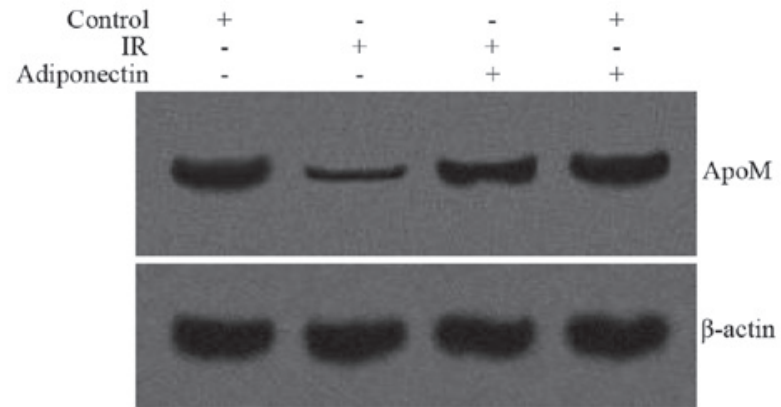

Figure 5. ApoM protein levels in HepG2 cells. Compared with the control group, ApoM protein levels were significantly decreased in the IR group. Following intervention, the ApoM protein levels were markedly increased in the IR group compared with the untreated IR group. There was no significant difference in ApoM protein levels between the control group and the adiponectin intervention group. $\beta$-actin was used as loading control. ApoM, apolipoprotein M; IR, insulin resistance.

mRNA expression levels were observed between the control group and the adiponectin intervention group ( $\mathrm{P}>0.05$; Fig. 6$)$.

Glucose consumption in Hep 2 cells. Glucose consumption in the IR group was significantly lower than the control group $(\mathrm{P}<0.05$; Fig. 7). This suggested that the glucose consumption capacity of the cells in the IR group was decreased, and the glucose uptake and utilization was impaired, which resulted in IR. Glucose consumption of the adiponectin intervention IR group was higher than the IR group $(\mathrm{P}<0.05$; Fig. 7) which suggested that adiponectin could increase the glucose consumption of IR HepG2 cells. There was no significant difference in glucose consumption between adiponectin intervention group and control group ( $\mathrm{P}>0.05$; Fig. 7).

\section{Discussion}

Obesity is closely associated with cardiovascular diseases, and is an independent risk factor for mortality and lower quality of life. Obesity is often correlated with disorders in carbohydrate and fat metabolism, the most significant of which is HDL-C deficiency, a risk factor for coronary heart disease. However, the mechanism by which HDL-C deficiency occurs in obesity remains unclear. Current evidence indicates that HDL-C deficiency could occur in obese patients because its particle size is small enough to easily penetrate the filter structure of the kidney and due to an overactive catabolism (24). ApoM is a recently discovered apolipoprotein that is closely associated with HDL-C. Increased HDL-C in plasma was observed in ApoM over-expressing mice whilst silencing of the ApoM gene resulted in a significant decrease in plasma HDL-C levels and a complete abolishment of pre- $\beta$-HDL, the primary form of HDL during its maturation as well as an important cholesterol receptor in somatic cells. The clearance rate of HDL from plasma and the uptake rate by somatic cells lacking ApoM were significantly increased (4). It has also been demonstrated that ApoM is the receptor for sphingosine-1-phosphate (S1P) in HDL particles with function primarily achieved via the ApoM/S1P axis (25-27). Furthermore, studies have identified that ApoM gene polymorphism is related to the metabolism of HDL in obese Korean male adults (28). The aforementioned 


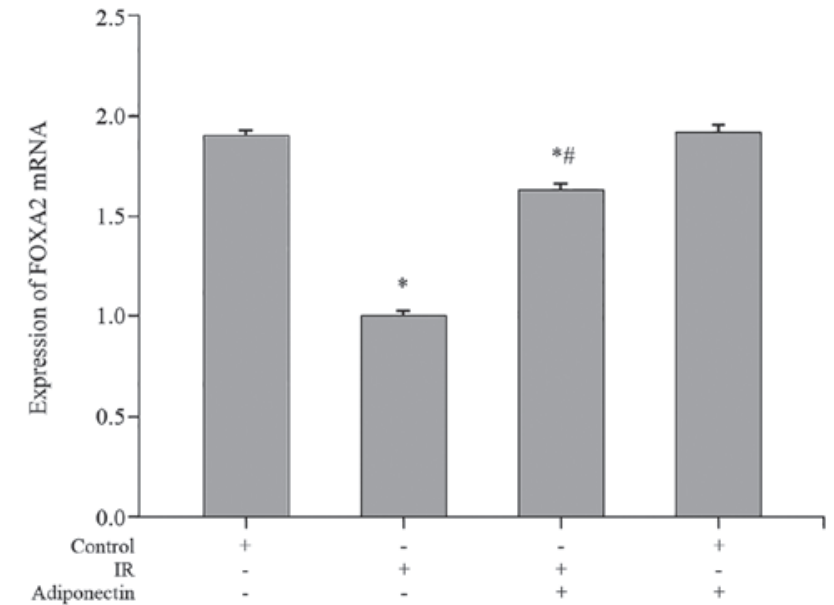

Figure 6. Foxa2 mRNA expression levels in HepG2 cells. Compared with the control group, the Foxa2 mRNA expression levels were significantly decreased in the IR group. Following treatment with adiponectin, the Foxa2 mRNA expression levels were significantly increased in the IR group compared with the untreated IR group. No significant difference in Foxa2 mRNA expression levels was observed between the control group and the adiponectin intervention group. ${ }^{*} \mathrm{P}<0.05$ vs. control; ${ }^{*} \mathrm{P}<0.05$ vs. IR group. Foxa2, forkhead box A2; IR, insulin resistance.

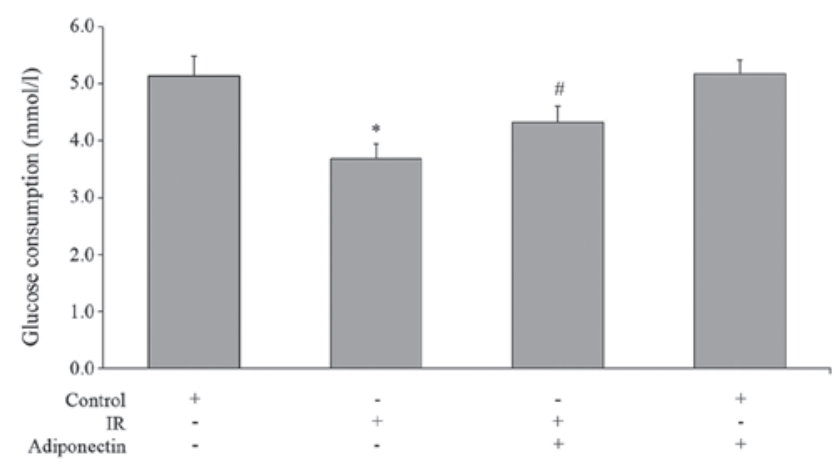

Figure 7. Glucose consumption in HepG2 cells. Glucose consumption in the IR group was significantly lower than the control group. Glucose consumption of the adiponectin intervention IR group was higher than the IR group. There was no significant difference in glucose consumption between the adiponectin intervention group and the control group. ${ }^{*} \mathrm{P}<0.05$ vs. control; ${ }^{\text {" }} \mathrm{P}<0.05$ vs. IR group. IR, insulin resistance.

findings demonstrate that ApoM is an important regulator in HDL-C metabolism. The present study demonstrated that mouse liver ApoM gene expression was significantly decreased in obese mice, which indicated that the decreased level of ApoM could be the underlying cause of HDL-C deficiency in obese patients. Since ApoM may affect the retro-trafficking of cholesterol via regulating the synthesis of pre- $\beta$-HDL, it was speculated that an abnormal ApoM level could lead to attenuated retro-trafficking of cholesterol in obese patients and subsequently accelerate the progression of obesity-related atherosclerosis.

In addition to its energy storage function, adipose tissue may also have some endocrinal functions and be closely associated with cardiovascular diseases via a series of adipocytokines (29). Both in vitro and in vivo studies have revealed that an adipocytokine, leptin, is an important regulator for ApoM expression (30,31). Studies have reported that the level of another adipocytokine, adiponectin, is positively correlated with HDL-C $(32,33)$. Therefore, the present study speculated that the metabolism of HDL might be related to adiponectin and the effect of adiponectin on ApoM was investigated.

Adiponectin is mainly secreted by adipose tissue and is currently the only known protective adipocytokine effectively alleviating IR and demonstrating anti-inflammatory and anti-atherosclerosis properties $(34,35)$. The present study demonstrated that obese mice exhibited an elevated plasma adiponectin level and ApoM expression was upregulated following adiponectin-treatment. Adiponectin intervention IR cells also exhibited marked upregulation of ApoM expression, which collectively suggested that the decreased ApoM expression in obesity was correlated with decreased adiponectin levels. Therefore, elevated adiponectin level may upregulate ApoM expression, indicating that adiponectin is another adipocytokine responsible for ApoM expression regulation. It is notable that the regulatory role of adiponectin on ApoM expression was only observed in IR HepG2 cells and not in normal HepG2 cells, suggesting that IR is required as a pre-condition for the regulatory effects of adiponectin. This was supported by the observation that adiponectin intervention in normal mice failed to upregulate ApoM expression.

Foxa2 is a crucial nuclear factor influencing the development of the liver and pancreas, and is also responsible for regulation of carbohydrate metabolism in hepatic and $\beta$ cells $(36,37)$. Wolfrum et al (38) confirmed the existence of a binding site for Foxa2 located 474 bp upstream of the promoter for ApoM gene, and demonstrated that ApoM expression was decreased in Foxa $2^{(+/)}$mice. In addition, injection of adenovirus encoding Foxa2 into mice could markedly elevate ApoM expression, which indicated that ApoM was a target gene of Foxa2. In the scenario of IR, insulin could inhibit transcription activity via the insulin/PI3K/AKT signaling pathway to downregulate the activity of Foxa2. Since hyperinsulinemia and IR usually accompany obesity, adiponectin is likely to indirectly regulate ApoM expression via ameliorating IR. This is supported by the observation of the present study that insulin sensitivity was increased in obese mice following adiponectin intervention. Adiponectin intervention in obese mice and IR HepG2 cells markedly upregulated Foxa 2 mRNA expression however it had minimal effect on normal mice, which indicated that adiponectin could indirectly upregulate Foxa2 expression via ameliorating IR, and therefore exert influence on ApoM.

In summary, ApoM gene expression in obese mice was markedly decreased, the mechanism of which may be associated with hypoadiponectinemia. Adiponectin promoted ApoM expression via upregulating Foxa 2 gene expression, with this function of adiponectin most likely achieved indirectly by ameliorating IR.

\section{Acknowledgements}

Not applicable.

\section{Funding}

The present study was supported by the Natural Science Foundation of Hunan Province, China (grant no. 14JJ7006), 
and the Science and Technology Innovation Planning Project of Hunan Province, China (grant no. 2017SK50104).

\section{Availability of data and materials}

The datasets used and/or analyzed during the current study are available from the corresponding author on reasonable request.

\section{Authors' contributions}

LY, TL, SPZ and SDZ conceived and designed the experiments. LY and TL performed the experiments. LY and TL collected and analyzed the data. LY and TL wrote the manuscript. All authors read and approved the final manuscript.

\section{Ethics approval and consent to participate}

Animal care and handling were completed in accordance with the National Institutes of Health Guide for the Care and Use of Laboratory Animals. All procedures were approved by the Ethics Committee of the Xiangya Hospital of Central South University (Changsha, China).

\section{Patient consent for publication}

Not applicable.

\section{Competing interests}

The authors declare that they have no competing interests.

\section{References}

1. Ravussin A and Bouchard C: Human genomics and obesity: Finding appropriate drug targets. Eur J Pharmaco1 410: 131-145, 2000.

2. Abbasi F, Brown BW Jr, Lamendola C, McLaughlin T and Reaven GM: Relationship between obesity, insulin resistance, and coronary heart disease risk. J Am Coll Cardiol 40: 937-943, 2002.

3. Sacks FM; Expert Group on HDL Cholesterol: The role of high-density lipoprotein (HDL) cholesterol in the prevention and treatment of coronary heart disease: Expert group recommendations. AM J cardiol 90: 139-143, 2002.

4. Wolfrum C, Poy MN and Stoffel M: Apolipoprotein M is required for prebeta-HDL formation and cholesterol efflux to HDL and protects against atherosclerosis. Nat Med 11: 418-422, 2005.

5. Everson SA, Goldbeng DE, Helmrich SP, Lakka TA, Lynch JW Kaplan GA and Salonen JT: Weight gain and the risk of developing insulin resistance syndrome. Diabetes Care 21: 1637-1643, 1998.

6. Caro JF: Clinical review 26: Insulin resistance in obese and nonobese man. J Clin Endocrinol Metab 73: 691-695, 1991.

7. Podskalny JM, Takeda S, Silverman RE, Tran D, Carpentier JL, Orci L and Gorden P: Insulin receptors and bioresponses in a human liver cell line (Hep G-2). Eur J Biochem 1502: 401-407, 1985.

8. Brillon DJ, Freidenberg GR, Henry RR and Olefsky JM: Mechanism of defective insulin-receptor kinase activity in NIDDM. Evidence for two receptor populations. Diabetes 38: 397-403, 1989.

9. Hari J and Roth RA: Defective internalization of insulin and its receptor in cells expressing mutated insulin receptors lacking kinase activity. J Biol Chem 262: 15341-15344, 1987.

10. Amatruda JM and Roncone AM: Normal hepatic insulin receptor autophosphorylation in nonketotic diabetes mellitus. Biochem Biophys Res Commun 129: 163-170, 1985.

11. Changgui L, Guang $\mathrm{N}$ and Jialun $\mathrm{C}$ : Establishing and identifing insulin resistant HepG2 cell line. Chin J Diabetes 7: 198-199, 1999.
12. Xie P, Liu ML, Gu YP, Lu J, Xu X, Zeng WM and Song HP Oestrogen improves glucose metabolism and insulin signal transduction in HepG2 cells. Clin Exp Pharmacol Physiol 30: 643-648, 2003

13. Zhang HJ, Ji BP, Chen G, Zhou F, Luo YC, Yu HQ, Gao FY, Zhang ZP and Li HY: A combination of grape seed-derived procyanidins and gypenosides alleviates insulin resistance in mice and HepG2 cells. J Food Sci 74: H1-H7, 2009.

14. Xie W, Wang W, Su H, Xing D, Pan Y and Du L: Effect of ethanolic extracts of Ananas comosus L. leaves on insulin sensitivity in rats and HepG2. Comp Biochem Physiol C Toxicol Pharmacol 143: 429-435, 2006.

15. Haluzik M, Parízková J and Haluzík MM: Adiponectin and its role in the obesity induced insulin resistance and related complications. Physiol Res 53: 123-129, 2004.

16. Yamashita R, Saito T, Satoh S, Kaburagi Y and Sekihara H: Effects of dehydroepiandrosterone on gluconeogenic enzymes and glucose uptake in human hepatoma cell line, HepG2. Endocr J 52: 727-733, 2005.

17. Chavez-Tapia NC, Rosso $\mathrm{N}$ and Tiribelli C: In vitro models for the study of non-alcoholic fatty liver disease. Curr Med Chem 18: 1079-1084, 2011.

18. Pullinger CR, North JD, Teng BB, Rifici VA, Ronhild de Brito AE and Scott $\mathrm{J}$ : The apolipoprotein $\mathrm{B}$ gene is constituently expressed in HepG2 cells: Regulation by oleic acid, albumin, and insulin, and measurement of the mRNA half life. J Lipid Res 30: 1065-1077, 1989.

19. Chao PM, Kuo YH, Lin YS, Chen CH, Chen SW and Kuo YH: The metabolic benefits of Polygonum hypoleucum Ohwi in HepG2 cells and Wistar rats under lipogenic stress. J Agric Food Chem 58: 5174-5180, 2010.

20. Liu Z, Kuang W, Xu X, Li D, Zhu W, Lan Z and Zhang X: Putative identification of components in Zengye Decoction and their effects on glucose consumption and lipogenesis in insulin-induced insulin-resistant HepG2 cells. J Chromatogr B Analyt Technol Biomed Life Sci 1073: 145-153, 2018.

21. Zheng X, Ke Y, Feng A, Yuan P, Zhou J, Yu Y, Wang X and Feng W: The Mechnism by which amentoflavone improves insulin resistance in HepG2 cells. Molecules 21: pii: E624, 2016.

22. Nie J, Chang Y, Li Y, Zhou Y, Qin J, Sun Z and Li H: Caffeic acid phenethyl ester (Propolis Extract) ameliorates insulin resistance by inhibiting $\mathrm{JNK}$ and $\mathrm{NF}-\kappa \mathrm{B}$ inflammatory pathways in diabetic mice and HepG2 cell models. J Agric Food Chem 65: 9041-9053, 2017.

23. Livak KJ and Schmittgen TD: Analysis of relative gene expression data using real-time quantitative PCR and the 2(-Delta Delta C(T)) method. Methods 25: 402-408, 2001.

24. Horowitz BS, Goldberg IJ, Merab J, Vanni TM, Ramakrishnan R and Ginsberg HN: Increased plasma and renal clearance of an exchangeable pool of apolipoprotein A-I in subjects with low levels of high density lipoprotein cholesterol. J Clin Invest 91: 1743-1752, 1993.

25. Ruiz M, Frej C, Holmér A, Guo LJ, Tran S and Dahlbäck B: High-density lipoprotein-associated apolipoprotein M limits endothelial inflammation by delivering Sphingosine-1-phosphate to the Sphingosine-1-phosphate receptor 1. Arterioscler Thromb Vasc Biol 37: 118-129, 2017.

26. Ruiz M, Okada H and Dahlbäck B: HDL-associated ApoM is anti-apoptotic by delivering sphingosine 1-phosphate to S1P1 \& S1P3 receptors on vascular endothelium. Lipids Health Dis 16: 36, 2017.

27. Frej C, Mendez AJ, Ruiz M, Castillo M, Hughes TA, Dahlbäck B and Goldberg RB: A shift in ApoM/S1P between HDL-Particles in women with type 1 diabetes mellitus is associated with impaired anti-Inflammatory effects of the ApoM/S1P complex. Arterioscler Thromb Vasc Biol 37: 1194-1205, 2017.

28. Lee M, Kim JI, Choi S, Jang Y and Sorn SR: The effect of apoM polymorphism associated with HDL metabolism on obese Korean Adults. J Nutrigenet Nutrigenomics 9: 306-317, 2016.

29. Calabro P and Yeh ET: Obesity, inflammation, and vascular disease: The role of the adipose tissue as an endocrine organ. Subcell Biochem 42: 63-91, 2007.

30. Xu N, Nilsson-Ehle P, Hurtig M and Ahrén B: Both leptin and leptin-receptor are essential for apolipoprotein $\mathrm{M}$ expression in vivo. Biochem Biophys Res Commun 321: 916-921, 2004.

31. Luo G, Hurtig M, Zhang X, Nilsson-Ehle P and Xu N: Leptin inhibits apolipoprotein $\mathrm{M}$ transcription and secretion in human hepatoma cell line, HepG2 cells. Biochim Biophys Acta 1734: 198-202, 2005 
32. Tsubakio-Yamamoto K, Sugimoto T, Nishida M, Okano R, Monden Y, Kitazume-Taneike R, Yamashita T, Nakaoka H, Kawase R, Yuasa-Kawase M, et al: Serum adiponectin level is correlated with the size of HDL and LDL particles determined by high performance liquid chromatography. Metabolism 61: 1763-1770, 2012.

33. Altinova AE, Toruner F, Bukan N, Yasar DG, Akturk M, Cakir N and Arslan M: Decreased plasma adiponectin is associated with insulin resistance and HDL cholesterol in overweight subjects. Endocr J 54: 221-226, 2007.

34. Zietz B, Herfaah H, Paul G, Ehling A, Müller-Ladner U, Schölmerich J and Schäffler A: Adiponectin represents and independent cardiovascular risk factor predicting serum HDL-C levels in type 2 diabetes. FEBS Lett 545: 103-104, 2003.

35. Dullaart RP, de Vries R, van Tol A and Sluiter WJ: Lower plasma adiponectin is a marker of increased intima-media thickness assiciated with type 2 diabetes mellitus and with male gender. Eur J Endocrinol 156: 387-394, 2007.
36. Sund NJ, Vatamaniuk MZ, Casey M, Ang SL, Magnuson MA, Stoffers DA, Matschinsky FM and Kaestner KH: Tissue-specific deletion of Foxa2 in pancreatic beta cells results in hyperinsulinemic hypoglycemia. Genes Dev 15: 1706-1715, 2001

37. Lee CS, Friedman JR, Fulmer JT and Kaestner KH: The initiation of liver development is dependent on Foxa transcription factors. Nature 435: 944-947, 2005.

38. Wolfrum C, Howell JJ, Ndungo E and Stoffel M: Foxa2 activity increases plasma high density lipoprotein levels by regulating apolipoprotein M. J Biol Chem 283: 16940-16949, 2008.

This work is licensed under a Creative Commons Attribution-NonCommercial-NoDerivatives 4.0 International (CC BY-NC-ND 4.0) License. 